\title{
Energy cooperation for throughput optimization based on save-then-transmit protocol in wireless communication system
}

\author{
Cui-Qin Dai $^{*}$, Fu-Jia Li ${ }^{1}$ and Markku Renfors ${ }^{2}$
}

\begin{abstract}
Green communication and energy saving have been a critical issue in modern wireless communication systems. The concepts of energy harvesting and energy transfer are recently receiving much attention in academic research field. In this paper, we study energy cooperation problems based on save-then-transmit protocol and propose two energy cooperation schemes for different system models: two-node communication model and three-node relay communication model. In both models, all of the nodes transmitting information have no fixed energy supplies and gain energy only via wireless energy harvesting from nature. Besides, these nodes also follow a save-then-transmit protocol. Namely, for each timeslot, a fraction (referred to as save-ratio) of time is devoted exclusively to energy harvesting while the remaining fraction is used for data transmission. In order to maximize the system throughput, energy transfer mechanism is introduced in our schemes, i.e., some nodes are permitted to share their harvested energy with other nodes by means of wireless energy transfer. Simulation results demonstrate that our proposed schemes can outperform both the schemes with half-allocate save-ratio and the schemes without energy transfer in terms of throughput performance, and also characterize the dependencies of system throughput, transferred energy, and save-ratio on energy harvesting rate.
\end{abstract}

Keywords: Energy harvesting; Energy transfer; Throughput optimization; Save-then-transmit protocol

\section{Introduction}

As the wireless communication technologies continue to evolve, the requirement for green communication and energy saving becomes more critical than ever. Especially, in some battery-powered wireless communication networks, the wireless devices do not have constant energy supplies for their mobility. The lifetime of a battery-powered network usually depends on the battery capacity. How to prolong the lifetime effectively and economically is still an open challenge. However, as a promising technique, energy harvesting has emerged and received significant attention in recent years [1-8]. Unlike the conventional scenario that wireless nodes are going to die if they exhaust their battery energy, the energy harvesting technique makes the node energy inexhaustible by means of recharging the battery from nature (solar cells, water mills, or mechanical vibration

\footnotetext{
* Correspondence: daicq@cqupt.edu.cn

${ }^{1}$ Chongqing Key Lab of Mobile Communication Technology, Chongqing

University of Posts and Telecommunications, Chongqing, China

Full list of author information is available at the end of the article
}

absorption devices, etc.). In this way, the energy harvesting cannot only prolong the network lifetime but also contribute to the green communication.

In $[2,3]$, the arrivals of energy harvesting packets were modeled as a stationary random process. Huang [2] modeled the throughput as a Poisson point process, so that the relationship between the throughput and the energy arrival rate provides useful insight into the tradeoff among the node density, encoding rate, and the amount of harvested energy. Tutuncuoglu and Yener [3] developed some optimal power policies, which demonstrated that the optimization problem is an instance of a utility maximization framework. Xu et al. [4] studied the downlink throughput of a coordinated multipoint enabled cellular network, where the base stations are powered by both the conventional grid and the harvested energy. In $[5,6]$, energy buffer and data buffer were adopted, with which sensor nodes employ management policies to optimize their throughput. A save-then-transmit protocol was proposed in $[7,8]$ to optimize the system performance by finding the optimal save-ratio. Luo et al. [7] derived the 
properties of the optimal save-ratio which minimizes outage probability, while [8] studied the achievable throughput optimization with regard to energy harvesting rate under deterministic case and stochastic case.

These references aforementioned mainly focused on the strategies of harvesting energy from nature to power wireless nodes. In addition, wireless energy transfer via radio signals is another solution to power the wireless nodes [9-17]. In [9-12], information transmission and energy transfer were processed simultaneously. Zhang and Ho $[9,10]$ studied a three-node network scenario, in which one receiver node harvests energy and the other receiver node decodes information separately from the broadcast signal of the transmitter node. And the optimal transmission strategy was derived to achieve various tradeoffs between maximal information rate and energy transfer. Ding et al. [11] studied a multiple sourcedestination pairs communication system under four different relaying strategies, i.e., based on different principles, the harvested energy from multiple sources is allocated among the different destinations by the relay node. Chen et al. [12] adopted a Nash equilibrium-based game theory for the multiple source-destination pairs communication system with relay interference channels. Where, both a pure network with a same relaying proto$\mathrm{col}$ and a hybrid network with mixed relaying protocols are discussed. Xiang and Tao [13] proposed a scheme considering the worst-case robust beam-forming design for the energy receiver and the information receiver with imperfect channel state information. In [14], a novel iterative resource allocation algorithm was proposed to maximize the energy efficiency of OFDM downlink systems. In [15-20], a switching mode between energy harvesting and data relaying was adopted for receiver node to solve the potential limitation that practical circuits are not yet able to harvest energy and decode information at the same time. In [15], an optimal mode switching rule was proposed to enable the transmitter node to replenish energy opportunistically from the unintended interference and/or the intended signal sent by the transmitter node, and optimizes the outage probability. Watfa et al. [16] proposed storing and forwarding techniques for multi-hop wireless energy transfer to improve energy transfer efficiency, i.e., the main power source prefer to transfer energy to the nearest nodes till they are fully charged. Park and Clerckx $[17,18]$ investigated joint wireless information and energy transfer in a twouser MIMO interference channel, where each receiver chooses to either decodes the incoming information signal or harvests energy. Krikidis et al. and Nasir et al. $[19,20]$ studied a three-node cooperative network scenario. In [19], a greedy switching policy was adopted where the relay node transmits information when its residual energy can support decoding at the destination node. In [20], a relay node was designed to separate information processing and energy harvesting by means of time switching and power splitting, and the achievable throughput was evaluated in both delay-limited and delay-tolerant transmission models.

All references discussed above only consider single aspect of energy management, i.e., energy harvesting or energy transfer. On the contrary, Gurakan et al. [21,22] took into account both energy harvesting from nature and energy transfer between nodes. This combination makes a better contribution to the performance improvement. In [21], a throughput optimization problem in two-way communication system scenario was investigated, where users can harvest energy from nature and can also transfer a portion of energy to each other. Gurakan et al. [22] extended the solution of [21] to adapt more network structures: relay channel and multiple access channel. Tutuncuoglu and Yener [23] considered a multi-access relay channel, where multiple transmitter nodes and one relay node execute the strategy of energy harvesting and energy transfer. And the sum rate maximization problem was decomposed into optimal energy transfer and optimal power allocation problems for simplicity.

In order to optimize network resource management, we research energy cooperation schemes by exploiting both energy harvesting and energy transfer strategies. Based on save-then-transmit protocol, we proposed two energy cooperation schemes for throughput optimization: two-node communication model with energy harvesting and energy transfer (TCM-EH-ET) and three-node relay communication model with energy harvesting and energy transfer (TRCM-EH-ET). Where, nodes in TCM-EH-ET model can harvest energy from nature and transfer energy to each other. In TRCM-EH-ET model, energy harvesting and energy transfer are only considered on source node and relay node. We assume that the nodes transmitting information have independent exogenous energy arrival processes to recharge their batteries and without considering energy expenditures on computation and sensing.

In this paper, we focus on finding appropriate save-ratio and energy transfer rates for throughput optimization in two-node communication model and three-node relay communication model, which, to the best of our knowledge, has not been studied yet. Unlike the existing energy harvesting and energy transfer models represented by energy queues [18-20], our proposed models depend on the allocation of time fraction in each timeslot. We prove the concavity of the achievable throughput and derive that the optimal save-ratio and energy transfer rates are functions of energy harvesting rate and channel coefficients for two given system models. We also characterize the dependencies of system throughput, transferred energy, and saveratio on energy harvesting rate through simulations. 


\section{TCM-EH-ET model}

As shown in Figure 1, in this section, we consider a selfpowered communication model consisting of two nodes: node 1 and node 2 . The two nodes have no fixed energy supplies and harvest energy exclusively from nature to recharge their batteries. For simplicity, we assume that, in each timeslot, the energy harvesting rates of node 1 and node 2 are constants notated as $x_{1}$ and $x_{2}\left(x_{1}>0\right.$, $x_{2}>0$ ). In this model, we define that the harvested energy will be transferred from node 1 to node 2 when $x_{1}>x_{2}$ and vice versa. When $x_{1}=x_{2}$, no energy transfer is permitted between the two nodes. On account of the self interference caused by the same physical channel, node 1 and node 2 exchange their information under a time division duplex communication model. We adopt the save-then-transmit protocol for each timeslot (with duration $T$ ), which is divided into three phases, as shown in Figure $2^{\mathrm{a}}$.

In the following, we give an example with the assumption of $x_{1}>x_{2}$.

\subsection{The first phase (energy harvesting and transfer)}

During time interval $(0, \rho T]$, node 1 and node 2 respectively harvest energy $E_{1}=\rho T x_{1}$ and $E_{2}=\rho T x_{2}$ from nature to recharge their batteries. Where, the save-ratio $\rho$ is subject to the constraint $0<\rho<1$. At the same time, energy $\Delta E=\rho T \delta$ ( $\delta$ is defined as energy transfer rate which is the power transferred from one node to the other node.) is transferred from one node harvesting more energy to the other node harvesting less energy. Obviously, the simultaneous charging and discharging of the node battery is not practical. And the model of simultaneous energy harvesting and transfer can be depictured as the Figure 3 below. The harvested energy $x_{1}$ of node 1 can be separated into two parts. One part of energy $x_{1}-\delta$ is used for battery charging, the other part of energy $\delta$ is directly transferred to node 2 . This model can avoid the simultaneous charging and discharging of the node battery.
According to the assumption of $x_{1}>x_{2}$, energy is transferred from node 1 to node 2 . After energy transfer process, the energy stored in node 1 and node 2 becomes

$$
\begin{aligned}
& E_{1}{ }^{\prime}=\rho T\left(x_{1}-\delta\right) \\
& E_{2}{ }^{\prime}=\rho T\left(x_{2}+\alpha \delta\right)
\end{aligned}
$$

where $\alpha$ is the energy transfer efficiency $(0<\alpha<1)$.

\subsection{The second phase (data transmission)}

During time interval $(\rho T, 0.5(1+\rho) T]$, node 1 transmits information to node 2 . The throughput of node 1 is modeled as

$$
\begin{aligned}
R_{12}(\rho, \delta)= & \frac{0.5(1-\rho) T}{T} \log _{2}\left[1+\frac{\left(x_{1}-\delta\right) \cdot \rho T \cdot h}{n_{1} \cdot 0.5(1-\rho) T}\right] \\
& =0.5(1-\rho) \log _{2}\left[1+\frac{\rho h\left(x_{1}-\delta\right)}{0.5(1-\rho)}\right]
\end{aligned}
$$

\subsection{The third phase (data transmission)}

During time interval $(0.5(1+\rho) T, T]$, node 2 transmits information to node 1 . The throughput of node 2 is modeled as

$$
\begin{aligned}
R_{21}(\rho, \delta) & =\frac{0.5(1-\rho) T}{T} \log _{2}\left[1+\frac{\left(x_{2}+\alpha \delta\right) \cdot \rho T \cdot h}{n_{2} \cdot 0.5(1-\rho) T}\right] \\
& =0.5(1-\rho) \log _{2}\left[1+\frac{\rho h\left(x_{2}+\alpha \delta\right)}{0.5(1-\rho)}\right]
\end{aligned}
$$

where $h$ denotes the channel coefficient between the two nodes ${ }^{\mathrm{b}}$. The noise power at node 1 and node 2 are $n_{1}$ and $n_{2}$ with unit value respectively.

Therefore, the throughput of two-node communication model is

$$
R_{\text {sum }}(\rho, \delta)=R_{12}(\rho, \delta)+R_{21}(\rho, \delta)
$$

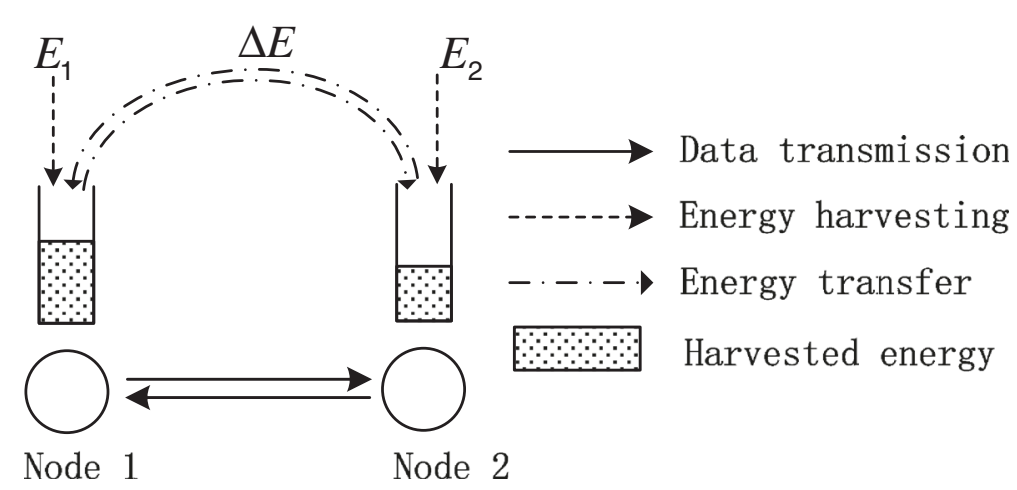

Figure 1 Two-node communication model with energy cooperation. 


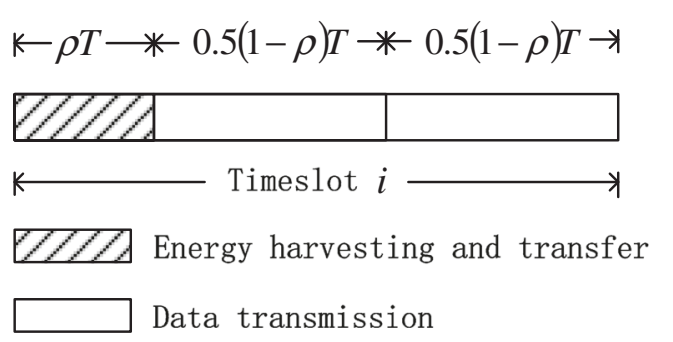

Figure 2 Save-then-transmit protocol with energy cooperation in timeslot $i$

\section{Throughput optimization for TCM-EH-ET}

In this section, we focus on the throughput optimization for TCM-EH-ET. The objective is

$$
\begin{array}{ll}
\max & R_{\text {sum }}(\rho, \delta)=0.5(1-\rho) \log _{2}\left[1+\frac{\rho h\left(x_{1}-\delta\right)}{0.5(1-\rho)}\right] \\
& +0.5(1-\rho) \log _{2}\left[1+\frac{\rho h\left(x_{2}+\alpha \delta\right)}{0.5(1-\rho)}\right] \\
\text { s.t. } & 0<\rho<1 \\
& 0 \leq \delta<x_{1}
\end{array}
$$

To solve this mathematic problem, several lemmas solving some specific equations are defined and employed in following sections.

\subsection{Lemma 1}

Assuming that continuous function $z=f(x, y)$ has first-order and second-order continuous partial derivatives at point $\left(x_{0}, y_{0}\right)$. And $f_{\mathrm{x}}\left(x_{0}, y_{0}\right)=0, f_{\mathrm{y}}\left(x_{0}, y_{0}\right)=0$. We define $f_{\mathrm{xx}}\left(x_{0}, y_{0}\right)=A, f_{\mathrm{xy}}\left(x_{0}, y_{0}\right)=B, f_{\mathrm{yy}}\left(x_{0}, y_{0}\right)=C$. If $A C-B^{2}>0$ and $A<0$, we can confirm that $f(x, y)$ is a concave function, which achieves maximal value at point $\left(x_{0}, y_{0}\right)$.

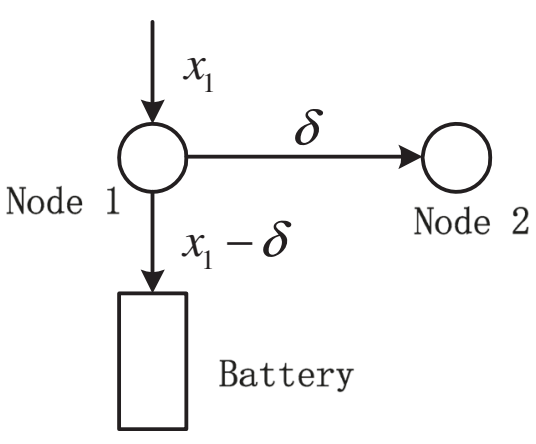

Figure 3 Simultaneous energy harvesting and energy transfer model.

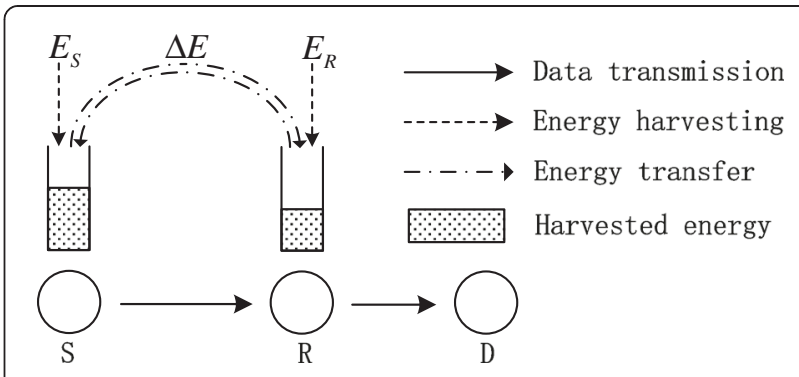

Figure $\mathbf{4}$ Three-node relay communication model with energy cooperation.

\subsection{Lemma 2}

We define that $R_{\text {sum }}(\rho, \delta)$ satisfy the conditions: $\frac{\partial R_{\text {sum }}}{\partial \rho} \mid$ $\left(\rho_{0}, \delta_{0}\right)=0,\left.\frac{\partial R_{\text {sum }}}{\partial \delta}\right|_{\left(\rho_{0}, \delta_{0}\right)}=0$. From lemma 1, we can confirm that $R_{\text {sum }}(\rho, \delta)$ is concave and achieves maximal value at point $\left(\rho_{0}, \delta_{0}\right)$ when $0<\rho<1$.

\subsection{Lemma 3}

The solution for the equation that has the form of $\ln x=$ $a x+b$ ( $\alpha$ and $b$ are constant) is given by

$$
x=-\frac{W\left(-a e^{\mathrm{b}}\right)}{a}
$$

where $W(\cdot)$ refers to the Lambert W function [24].

The proof of lemma 1 can be found in [25]. And the proof of lemma 2 and lemma 3 can be found in Appendix.

The throughput optimization strategy for TCM-EHET is demonstrated as follows. To simplify the equations, we introduce a new parameter

$$
Y=\frac{0.5(1-\rho)}{\rho h\left(x_{1}-\delta\right)-0.5(1-\rho)}
$$

From function $\left.\frac{\partial R_{\text {sum }}}{\partial \rho}\right|_{\left(\rho_{0}, \delta_{0}\right)}=0$ and $\left.\frac{\partial R_{\text {sum }}}{\partial \delta}\right|_{\left(\rho_{0}, \delta_{0}\right)}=0$, we can get

$$
\begin{gathered}
\rho h\left(x_{2}+\alpha \delta\right)+0.5(1-\rho)=\alpha \cdot\left[\rho h\left(x_{1}-\delta\right)+0.5(1-\rho)\right] \\
\ln \left[\frac{\rho h\left(x_{1}-\delta\right)+0.5(1-\rho)}{0.5(1-\rho)}\right]+\ln \left[\frac{\rho h\left(x_{2}+\alpha \delta\right)+0.5(1-\rho)}{0.5(1-\rho)}\right] \\
=\frac{h\left(x_{1}-\delta\right)}{\rho h\left(x_{1}-\delta\right)+0.5(1-\rho)}+\frac{h\left(x_{2}+\alpha \delta\right)}{\rho h\left(x_{2}+\alpha \delta\right)+0.5(1-\rho)}
\end{gathered}
$$




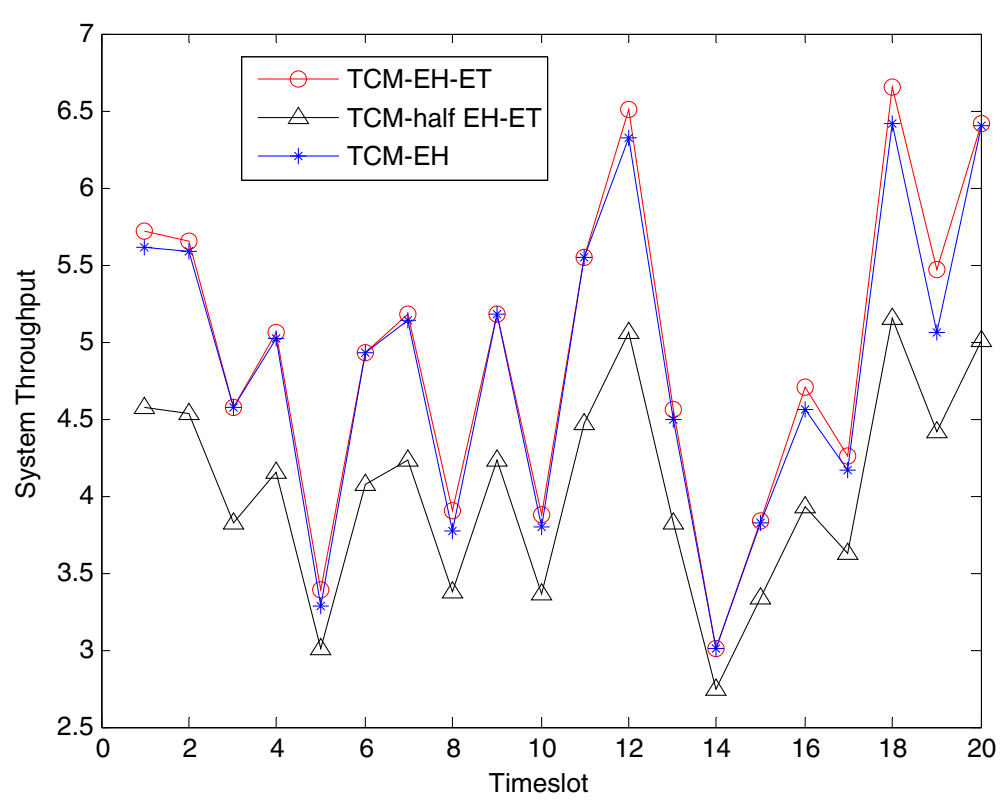

Figure 5 Simulation result with $x_{1}$ and $x_{2}$ generated from $\Gamma(5,5)$ and $\Gamma(5,5)$.

From Equations (8) and (9), we can get

$$
\delta=\frac{x_{1}(Y-\alpha)+x_{2}(1-Y)}{Y(1+\alpha)-2 \alpha}
$$

Put Equation (9) into Equation (10), we can get

$$
\begin{gathered}
\ln \left[\frac{\rho h\left(x_{1}-\delta\right)+0.5(1-\rho)}{0.5(1-\rho)}\right]+\ln \left[\alpha \cdot \frac{\rho h\left(x_{1}-\delta\right)+0.5(1-\rho)}{0.5(1-\rho)}\right] \\
=\frac{0.5(1-\rho)}{\rho h\left(x_{1}-\delta\right)+0.5(1-\rho)}\left(2 h x_{1}+\frac{2}{\alpha} h x_{2}-1-\frac{1}{\alpha}\right)+2 \\
\Rightarrow \ln Y=-\left(h x_{1}+\frac{1}{\alpha} h x_{2}-0.5-\frac{1}{2 \alpha}\right) \cdot Y+\ln \sqrt{\alpha}-1
\end{gathered}
$$

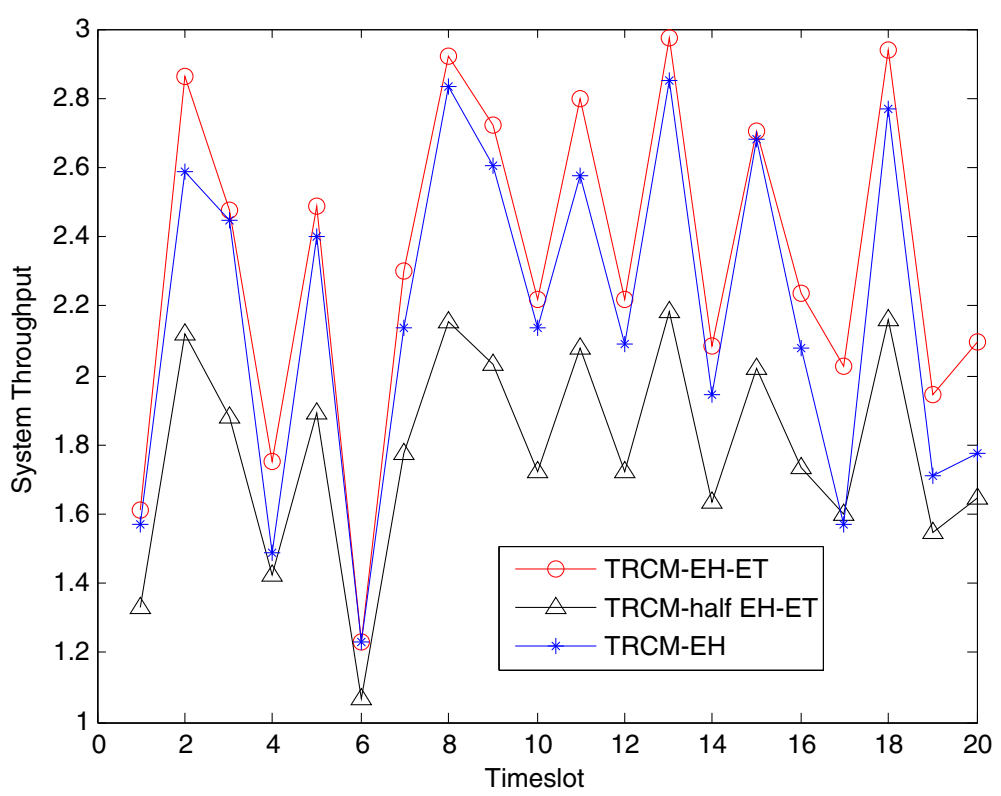

Figure 6 Simulation result with $y_{1}$ and $y_{2}$ generated from $\Gamma(5,5)$ and $\Gamma(5,5)$. 


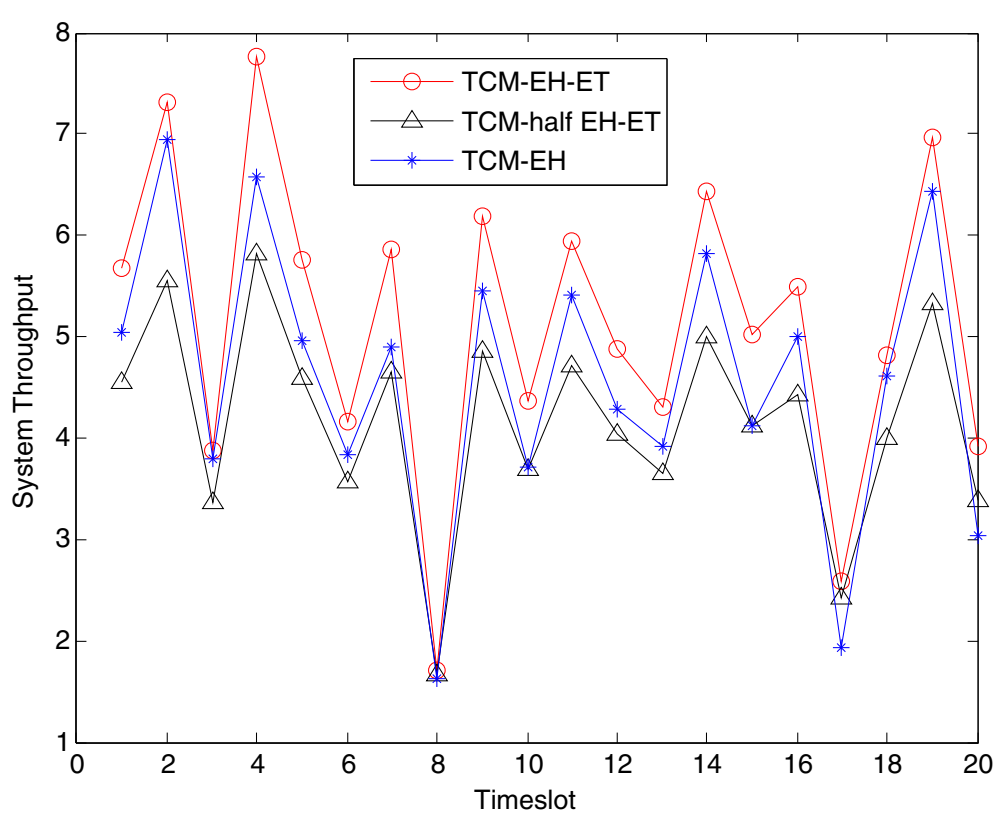

Figure 7 Simulation result with $x_{1}$ and $x_{2}$ generated from $\Gamma(8,8)$ and $\Gamma(3,3)$.

According to lemma 3, we can get the value of $Y$ from Equation (12)

$$
Y=\frac{W\left[\left(h x_{1}+\frac{1}{\alpha} h x_{2}-0.5-\frac{1}{2 \alpha}\right) \cdot \frac{\sqrt{\alpha}}{e}\right]}{h x_{1}+\frac{1}{\alpha} h x_{2}-0.5-\frac{1}{2 \alpha}}
$$

$$
\left\{\begin{array}{c}
\delta_{0}=\frac{x_{2}(Y-\alpha)+x_{1}(1-Y)}{Y(1+\alpha)-2 \alpha} \\
\rho_{0}=\frac{0.5(1-Y)}{h\left(x_{1}-\delta_{0}\right) Y-0.5 Y+0.5}
\end{array}\right.
$$

Therefore, according to lemma 2, we can get the solu- Similarly, in case of $x_{1}<x_{2}$, the solution of $\max$ tion of $\max R_{\text {sum }}(\rho, \delta)$ from Equations (8), (11), and (13) $\quad R_{\text {sum }}(\rho, \delta)$ is

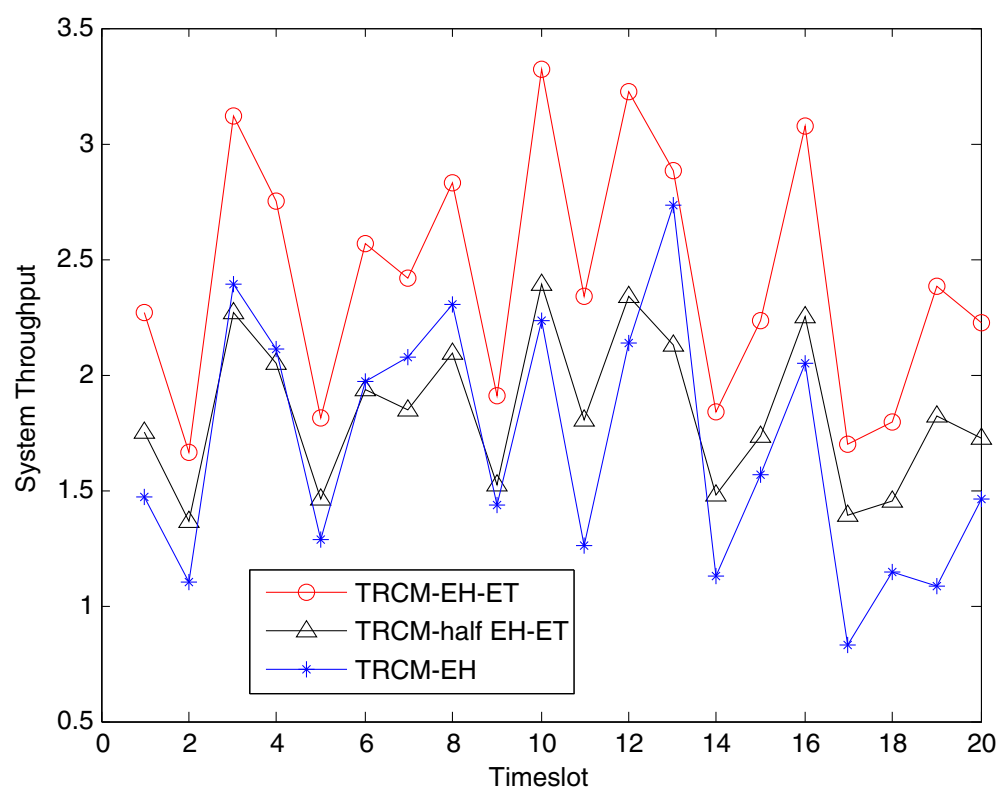

Figure 8 Simulation result with $y_{1}$ and $y_{2}$ generated from $\Gamma(8,8)$ and $\Gamma(3,3)$. 


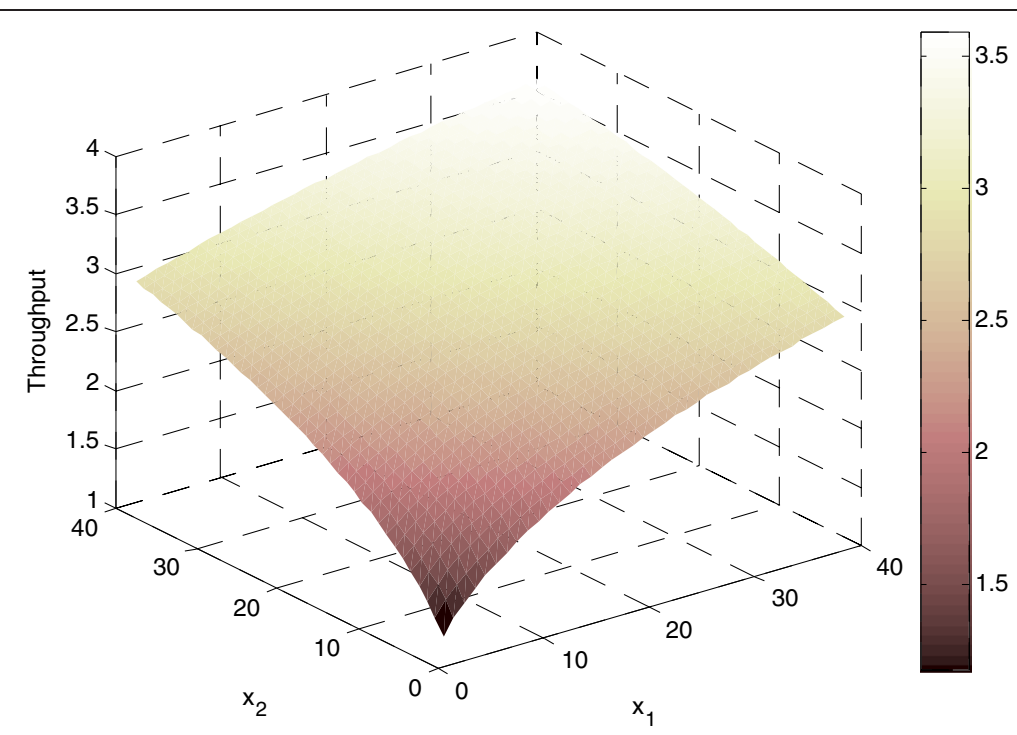

Figure 9 Throughput versus energy harvesting rate $x_{1}$ and $x_{2}$.

$$
\left\{\begin{array}{c}
\delta_{0}=\frac{x_{2}(Y-\alpha)+x_{1}(1-Y)}{Y(1+\alpha)-2 \alpha} \\
\rho_{0}=\frac{0.5(1-Y)}{h\left(x_{2}-\delta_{0}\right) Y-0.5 Y+0.5}
\end{array}\right.
$$

where the value of $Y$ turns into

$$
Y=\frac{W\left[\left(h x_{2}+\frac{1}{\alpha} h x_{1}-0.5-\frac{1}{2 \alpha}\right) \cdot \frac{\sqrt{\alpha}}{e}\right]}{h x_{2}+\frac{1}{\alpha} h x_{1}-0.5-\frac{1}{2 \alpha}}
$$

When $x_{1}=x_{2}$, energy transfer is not permitted between nodes, i.e., $\Delta E=0$. The solution can be derived as

$$
\left\{\begin{array}{c}
\delta_{0}=0 \\
\rho_{0}=\frac{2 x_{1} h-1-W\left(\frac{2 x_{1} h-1}{e}\right)}{\left(2 x_{1} h-1\right)\left[W\left(\frac{2 x_{1} h-1}{e}\right)+1\right]}
\end{array}\right.
$$

\section{TRCM-EH-ER model}

As shown in Figure 4, in this section, we consider a selfpowered three-node relay communication model consisting of one source node $S$, one relay node $R$, and one destination node $D . S$ and $R$ have no fixed energy supplies and harvest energy exclusively from nature to recharge

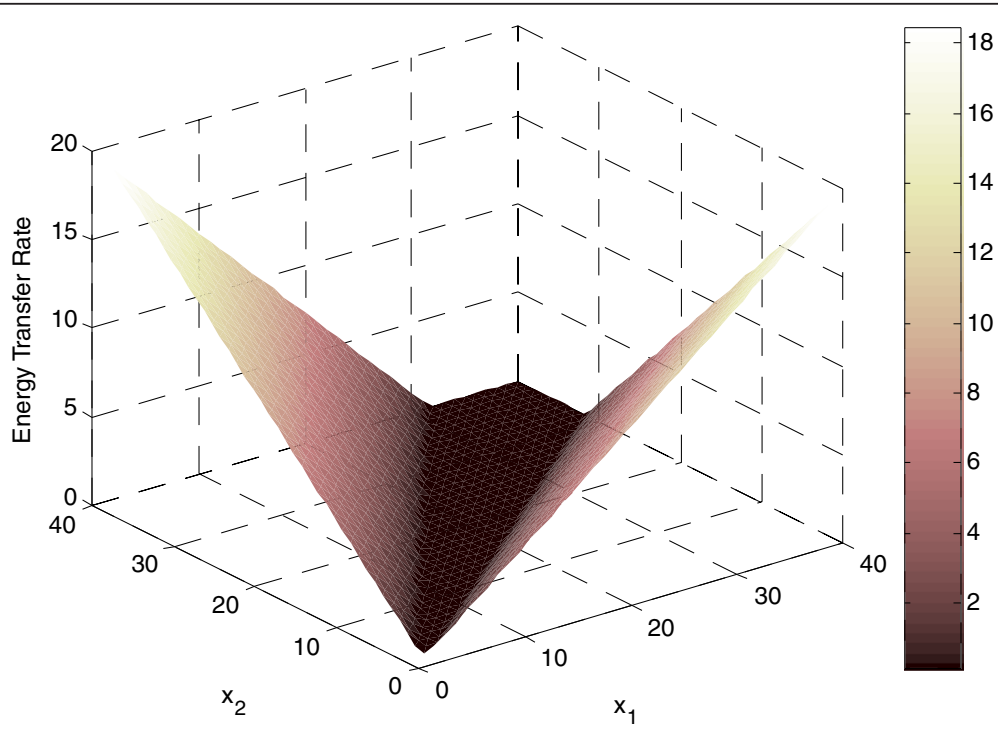

Figure 10 Energy transfer rate versus energy harvesting rate $x_{1}$ and $x_{2}$. 


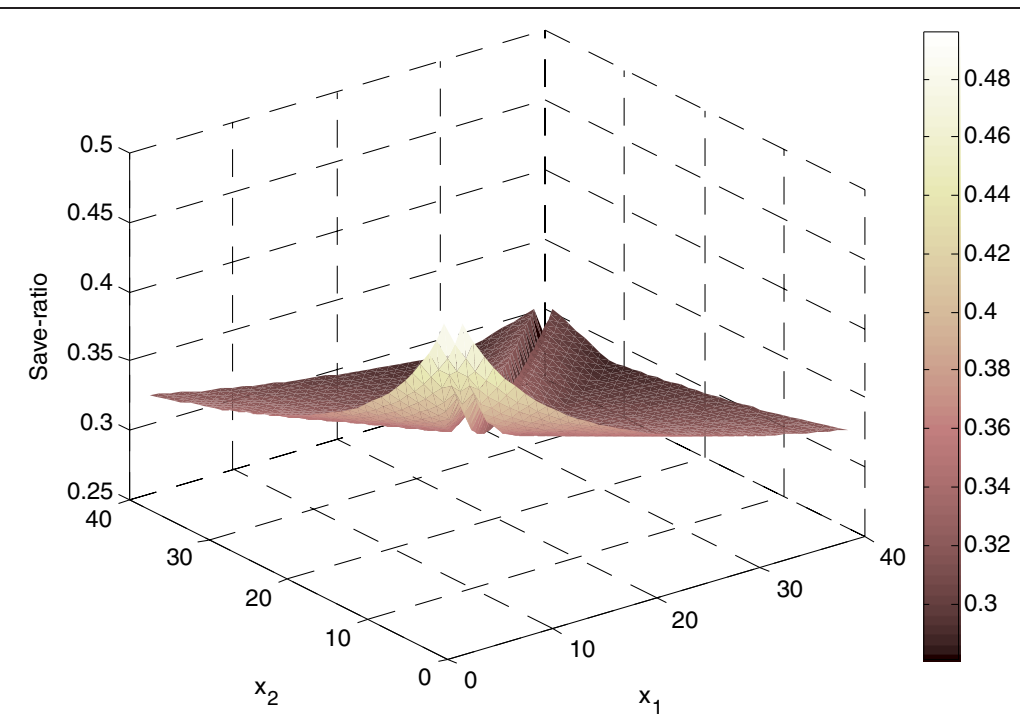

Figure 11 Save-ratio versus energy harvesting rate $x_{1}$ and $x_{2}$.

their batteries. We assume that, in each timeslot, the energy harvesting rates of $S$ and $R$ are constants notated as $y_{1}$ and $y_{2}\left(y_{1}>0, y_{2}>0\right)$. To maximize the system throughput, energy transfer between $S$ and $R$ is permitted.

For the three-node relay communication model, there is a constraint in terms of causality: The relay transmits data coming from the source. Therefore, the energy policies of the source and the relay need to satisfy the data causality constraint $R_{\mathrm{rd}} \leq R_{\mathrm{sr}}$. Where, $R_{\mathrm{sr}}$ and $R_{\mathrm{rd}}$ refer to the throughput of $S$ and $R$, respectively. Unlike the two-node communication model presented in section 2 , the optimization problem of three-node relay communication model is to maximize the throughput $R_{\mathrm{rd}}$. Namely, we have $R_{\mathrm{rd}}=R_{\mathrm{sr}}$ when the system throughput is optimized.

Similar to TCM-EH-ET, we also adopt the save-thentransmit protocol in TRCM-EH-ET ${ }^{c}$.

\subsection{The first phase (energy harvesting and transfer)}

During time interval $(0, \rho T], S$ and $R$ respectively harvest energy $E_{1}=\rho T y_{1}$ and $E_{2}=\rho T y_{2}$ from nature to recharge their batteries. At the same time, energy $\Delta E=\rho T \delta$ is transferred between $S$ and $R$.

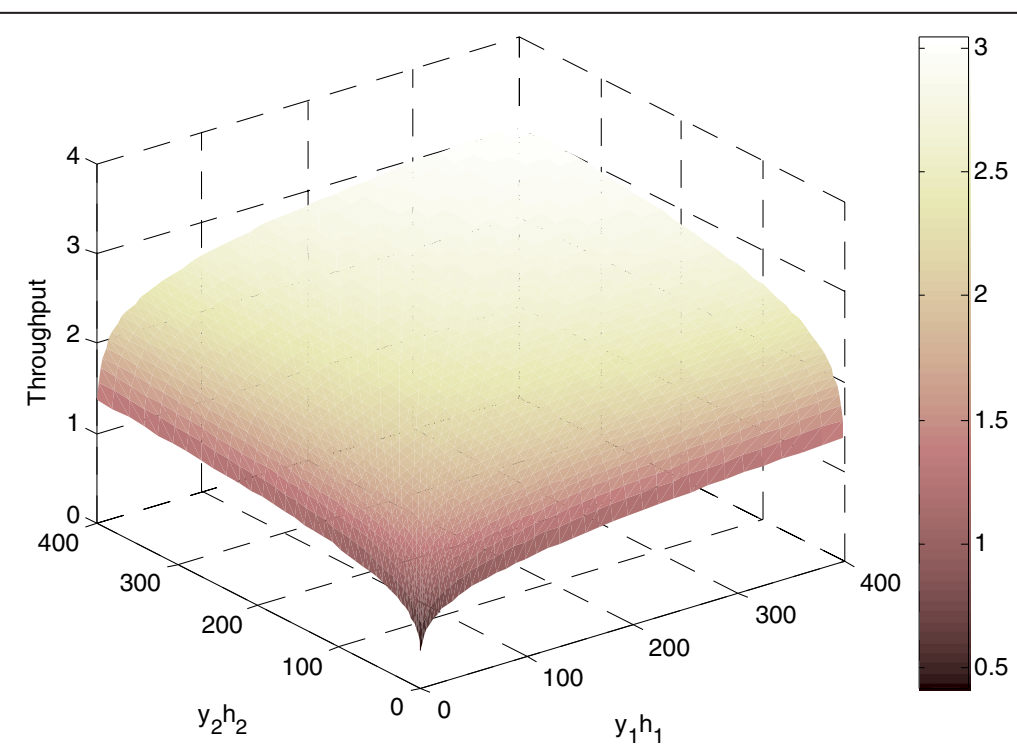

Figure 12 Throughput versus value of $y_{1} h_{1}$ and $y_{2} h_{2}$. 


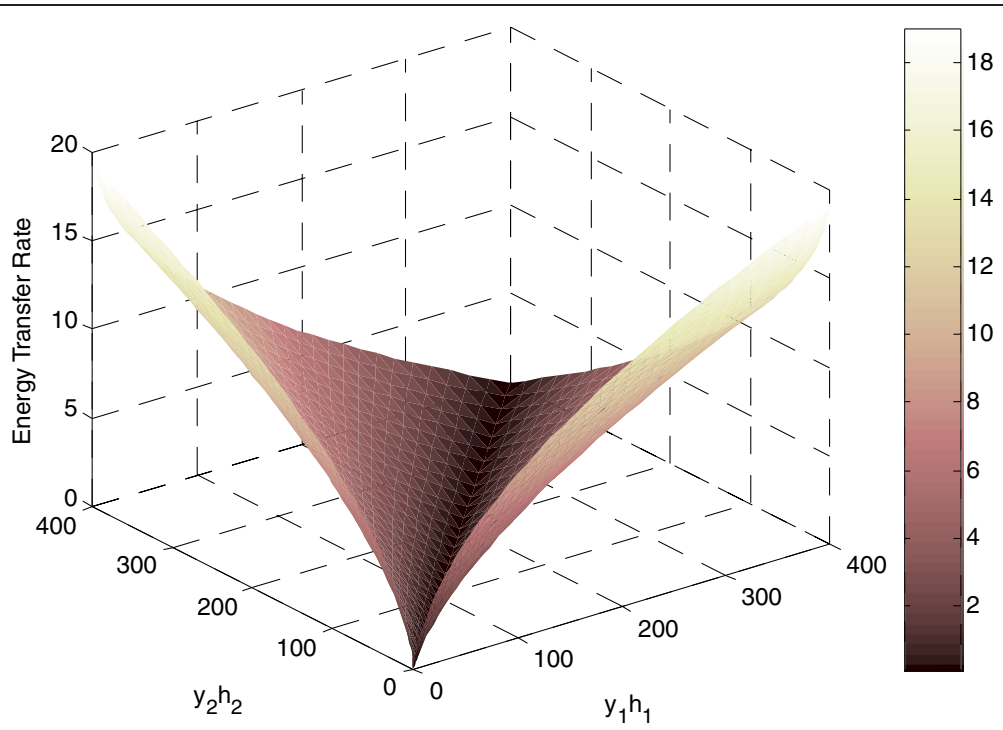

Figure 13 Energy transfer rate versus value of $y_{1} h_{1}$ and $y_{2} h_{2}$.

$$
\begin{aligned}
\bar{R}_{s r} & =\frac{0.5(1-\rho) T}{T} \log _{2}\left[1+\frac{y_{1} \cdot \rho T \cdot h_{1}}{0.5(1-\rho) T \cdot n_{s r}}\right] \\
& =0.5(1-\rho) \log _{2}\left[1+\frac{y_{1} h_{1} \rho}{0.5(1-\rho) n_{s r}}\right] \\
\bar{R}_{r d} & =\frac{0.5(1-\rho) T}{T} \log _{2}\left[1+\frac{y_{2} \cdot \rho T \cdot h_{2}}{0.5(1-\rho) T \cdot n_{r d}}\right] \\
& =0.5(1-\rho) \log _{2}\left[1+\frac{h_{2} y_{2} \rho}{0.5(1-\rho) n_{r d}}\right]
\end{aligned}
$$

Where $\bar{R}_{\mathrm{sr}}$ and $\bar{R}_{\mathrm{rd}}$ are the throughput of $S$ and $R$ before energy transfer process. $h_{1}$ and $h_{2}$ denote the channel coefficients for the source-to-relay and relay-todestination channels, respectively. $n_{\mathrm{sr}}$ and $n_{\mathrm{rd}}$ are the noise power with unit value at $S$ and $R$, respectively.

According to their different energy harvesting rate and channel coefficients, the energy transfer policy has three different methods.

In the following, we give an example with the assumption of $\bar{R}_{\text {rd }}<\bar{R}_{\text {sr }}\left(y_{2} h_{2}<y_{1} h_{1}\right)$. Thus, energy $\Delta E$ is transferred from $S$ to $R$. Then the energy stored in $S$ and $R$ turns into

$$
E_{S}{ }^{\prime}=\rho T\left(y_{1}-\delta\right)
$$

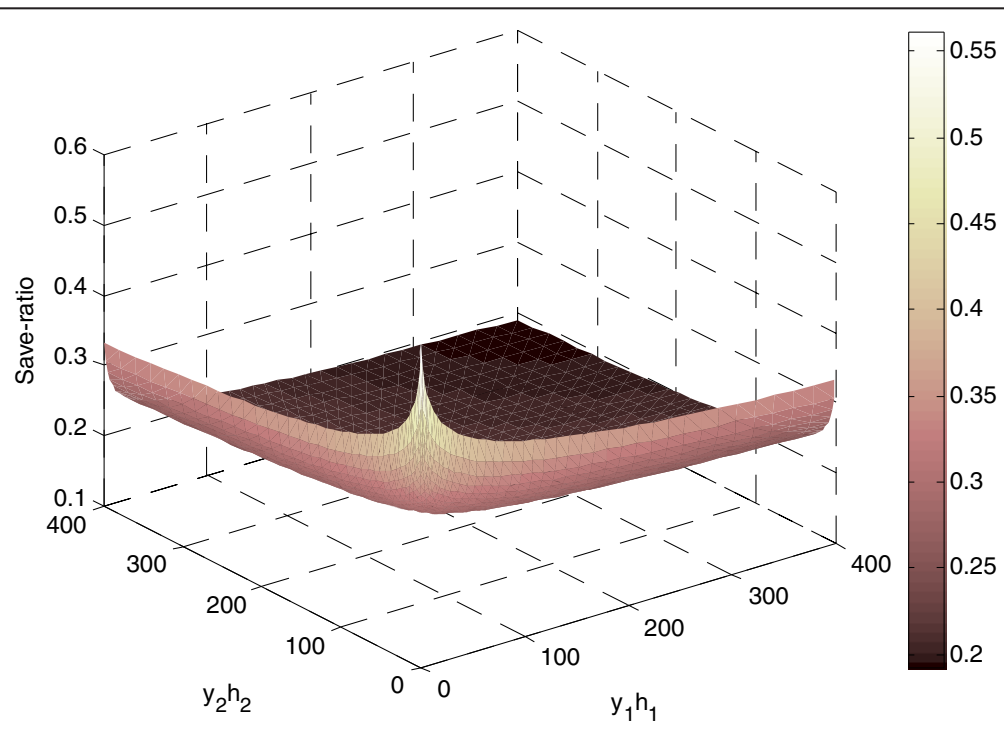

Figure 14 Save-ratio versus value of $y_{1} h_{1}$ and $y_{2} h_{2}$. 


$$
E_{R}^{\prime}=\rho T\left(y_{2}+\alpha \delta\right)
$$

\subsection{The second phase (data transmission)}

During time interval $(\rho T, 0.5(1+\rho) T], S$ transmits information to $R$, the throughput of $S$ is modeled as

$$
\begin{aligned}
R_{\mathrm{sr}}(\rho, \delta)= & \frac{0.5(1-\rho) T}{T} \log _{2}\left[1+\frac{\left(y_{1}-\delta\right) \cdot \rho T \cdot h_{1}}{0.5(1-\rho) T \cdot n_{\mathrm{sr}}}\right] \\
& =0.5(1-\rho) \cdot \log _{2}\left[1+\frac{\rho h_{1}\left(y_{1}-\delta\right)}{0.5(1-\rho)}\right]
\end{aligned}
$$

\subsection{The third phase (data transmission)}

During time interval $(0.5(1+\rho) T, T], R$ forwards information from $S$ to $D$, the throughput of $R$ is modeled as

$$
\begin{aligned}
R_{\mathrm{rd}}(\rho, \delta)= & \frac{0.5(1-\rho) T}{T} \log _{2}\left[1+\frac{\left(y_{2}+\alpha \delta\right) \cdot \rho T \cdot h_{2}}{0.5(1-\rho) T \cdot n_{\mathrm{rd}}}\right] \\
& =0.5(1-\rho) \cdot \log _{2}\left[1+\frac{\rho h_{2}\left(y_{2}+\alpha \delta\right)}{0.5(1-\rho)}\right]
\end{aligned}
$$

Where $R_{\mathrm{sr}}(\rho, \delta)$ and $R_{\mathrm{rd}}(\rho, \delta)$ are the throughput of $S$ and $R$ after energy transfer.

\section{Throughput optimization for TRCM-EH-ET}

In this section, we focus on the throughput optimization for TRCM-EH-ET. The objective is

$$
\begin{aligned}
\max & R_{\mathrm{rd}}(\rho, \delta)=0.5(1-\rho) \cdot \log _{2}\left[1+\frac{\rho h_{2}\left(y_{2}+\alpha \delta\right)}{0.5(1-\rho)}\right] \\
\text { s.t. } & 0<\rho<1 \\
& 0 \leq \delta<y_{1} \\
& R_{\mathrm{rd}}(\rho, \delta)=R_{\mathrm{sr}}(\rho, \delta)
\end{aligned}
$$

On account of the restriction above, we utilize the Lagrange multiplier method to solve the problem. The corresponding destination function is modeled as

$$
\begin{aligned}
L(\rho, \delta)= & 0.5(1-\rho) \cdot \log _{2}\left[1+\frac{\rho h_{2}\left(y_{2}+\alpha \delta\right)}{0.5(1-\rho)}\right]+ \\
& \lambda\left\{0.5(1-\rho) \cdot \log _{2}\left[1+\frac{\rho h_{1}\left(y_{1}-\delta\right)}{0.5(1-\rho)}\right]\right. \\
& \left.-0.5(1-\rho) \cdot \log _{2}\left[1+\frac{\rho h_{2}\left(y_{2}+\alpha \delta\right)}{0.5(1-\rho)}\right]\right\}
\end{aligned}
$$

The first-order continuous partial derivative of $L(\rho, \delta)$ can be expressed as

$$
\begin{aligned}
\frac{\partial L}{\partial \delta}= & 0.5(1-\lambda) \frac{\alpha \rho h_{2}(1-\rho)}{\ln 2 \cdot\left[\rho h_{2}\left(y_{2}+\alpha \delta\right)+0.5(1-\rho)\right]} \\
& -0.5 \lambda \frac{\rho h_{1}(1-\rho)}{\ln 2 \cdot\left[\rho h_{1}\left(y_{1}-\delta\right)+0.5(1-\rho)\right]}
\end{aligned}
$$

$$
\begin{aligned}
\frac{\partial L}{\partial \rho}= & 0.5(\lambda-1) \log _{2}\left[1+\frac{\rho h_{2}\left(y_{2}+\alpha \delta\right)}{0.5(1-\rho)}\right] \\
& -0.5 \lambda \log _{2}\left[1+\frac{\rho h_{1}\left(y_{1}-\delta\right)}{0.5(1-\rho)}\right] \\
& -0.5(\lambda-1) \frac{h_{2}\left(y_{2}+\alpha \delta\right)}{\ln 2 \cdot\left[\rho h_{2}\left(y_{2}+\alpha \delta\right)+0.5(1-\rho)\right]} \\
& +0.5 \lambda \frac{h_{1}\left(y_{1}-\delta\right)}{\ln 2 \cdot\left[\rho h_{1}\left(y_{1}-\delta\right)+0.5(1-\rho)\right]}
\end{aligned}
$$

$$
\begin{aligned}
\frac{\partial L}{\partial \lambda}= & 0.5(1-\rho) \cdot \log _{2}\left[1+\frac{\rho h_{1}\left(y_{1}-\delta\right)}{0.5(1-\rho)}\right] \\
& -0.5(1-\rho) \cdot \log _{2}\left[1+\frac{\rho h_{2}\left(y_{2}+\alpha \delta\right)}{0.5(1-\rho)}\right]
\end{aligned}
$$

From $\left.\frac{\partial L}{\partial \rho}\right|_{\left(\rho_{0}, \delta_{0}\right)}=0,\left.\frac{\partial L}{\partial \delta}\right|_{\left(\rho_{0}, \delta_{0}\right)}=0$ and $\left.\frac{\partial L}{\partial \lambda}\right|_{\left(\rho_{0}, \delta_{0}\right)}$ $=0$, the solution to Equation (25) can be derived as

$$
\left\{\begin{array}{l}
\delta_{0}=\frac{h_{1} y_{1}-h_{2} y_{2}}{h_{1}+\alpha h_{2}} \\
\rho_{0}=\frac{h_{2}\left(y_{2}+\alpha \delta_{0}\right)-0.5-0.5 W\left[\left(2 h_{2}\left(y_{2}+\alpha \delta_{0}\right)-1\right) \cdot e^{-1}\right]}{\left[h_{2}\left(y_{2}+\alpha \delta_{0}\right)-0.5\right] \cdot\left\{W\left[\left(2 h_{2}\left(y_{2}+\alpha \delta_{0}\right)-1\right) \cdot e^{-1}\right]+1\right\}}
\end{array}\right.
$$

Similarly, in case of $\bar{R}_{\text {rd }}>\bar{R}_{\text {sr }}\left(y_{2} h_{2}>y_{1} h_{1}\right)$, energy $\Delta E$ is transferred from $R$ to $S$. Then the energy stored in $S$ and $R$ turns into

$$
\begin{aligned}
& E_{S}{ }^{\prime}=\rho T\left(y_{1}+\alpha \delta\right) \\
& E_{R}{ }^{\prime}=\rho T\left(y_{2}-\delta\right)
\end{aligned}
$$

The corresponding destination function is modeled as

$$
\begin{aligned}
& L(\rho, \delta)=\mu \cdot \log _{2}\left[1+\frac{\rho h_{2}\left(y_{2}-\delta\right)}{\mu}\right]+ \\
& \lambda\left\{\mu \cdot \log _{2}\left[1+\frac{\rho h_{2}\left(y_{2}-\delta\right)}{\mu}\right]-\mu \cdot \log _{2}\left[1+\frac{\rho h_{2}\left(y_{1}+\alpha \delta\right)}{\mu}\right]\right\}
\end{aligned}
$$

The solution to Equation (32) is

$$
\left\{\begin{array}{c}
\delta_{0}=\frac{h_{2} y_{2}-h_{1} y_{1}}{\alpha h_{1}+h_{2}} \\
\rho_{0}=\frac{h_{2}\left(y_{2}-\delta_{0}\right)-0.5-0.5 W\left[\left(2 h_{2}\left(y_{2}-\delta_{0}\right)-1\right) \cdot e^{-1}\right]}{\left[h_{2}\left(y_{2}-\delta_{0}\right)-0.5\right] \cdot\left\{W\left[\left(2 h_{2}\left(y_{2}-\delta_{0}\right)-1\right) \cdot e^{-1}\right]+1\right\}}
\end{array}\right.
$$

When $\quad \bar{R}_{\text {rd }}=\bar{R}_{\text {sr }}\left(y_{2} h_{2}=y_{1} h_{1}\right)$, energy will not be transferred between $R$ and $S$, i.e., $\Delta E=0$. 
The corresponding destination function is modeled as

$$
\begin{aligned}
& L(\rho, \delta)=\mu \cdot \log _{2}\left[1+\frac{\rho h_{2} y_{2}}{\mu}\right]+ \\
& \lambda\left\{\mu \cdot \log _{2}\left[1+\frac{\rho h_{2} y_{2}}{\mu}\right]-\mu \cdot \log _{2}\left[1+\frac{\rho h_{2} y_{1}}{\mu}\right]\right\}
\end{aligned}
$$

Similarly, the solution to Equation (34) is

$$
\left\{\begin{array}{c}
\delta_{0}=0 \\
\rho_{0}=\frac{h_{2} y_{2}-0.5-0.5 W\left[\left(2 h_{2} y_{2}-1\right) \cdot e^{-1}\right]}{\left(h_{2} y_{2}-0.5\right) \cdot\left\{W\left[\left(2 h_{2} y_{2}-1\right) \cdot e^{-1}\right]+1\right\}}
\end{array}\right.
$$

\section{Simulation analysis}

In this section, our simulation results are based on the following premises:

- The channel coefficients are modeled as Rayleighdistributed in stochastic case and remain unchanged in each timeslot.

- Energy harvesting rate is Gamma-distributed in stochastic case, because Gamma distribution can model many positive random variables [26] and remain unchanged in each timeslot.

- The noise at each node is assumed as zero-mean and unit-variance.

- The energy transfer efficiency $\alpha$ is equal to 0.8 .

We suppose that $x$ is subject to a Gamma distribution, i.e., $x \sim \Gamma(k, \theta)$. Where $k(k>0), \theta(\theta>0)$, and $\Gamma($.$) refers$ to the shape parameter, the scale parameter, and the gamma function, respectively. Thus, 20 samples of energy harvesting rates are generated for our simulation.

In this paper, we simulate three schemes in two-node communication model, i.e., TCM-EH-ET, TCM-half EHET (the save-ratio $\rho=0.5$ ), and TCM-EH schemes (without energy transfer). We also simulate three schemes in three-node relay communication model, i.e., TRCM-EHET, TRCM-half EH-ER (the save-ratio $\rho=0.5$ ), and TRCM-EH schemes (without energy transfer).

Simulation results (from Figures 5, 6, 7, and 8) show that the throughputs of both TCM-EH-ET and TRCMEH-ET outperform all of their counterparts.

In Figure 5, we notice that, in two-node communication model, the throughput of TCM-EH-ET is very close to that of TCM-EH. That is because the energy transfer depends on the relationship between energy harvesting rates $\left(x_{1}\right.$ and $\left.x_{2}\right)$. Since $x_{1}$ and $x_{2}$ are generated by the same Gamma distribution $\Gamma(5,5), x_{1}$ is very close to $x_{2}$. Thus, the possibility of energy transfer decreases. In addition, the throughput of TCM-half EH-ET is obviously lower than that of TCM-EH-ET, the reason is that the optimal save-ratio $\rho$ dynamically changes according to energy harvesting rate $\left(x_{1}\right.$ and $\left.x_{2}\right)$.
In Figure 6, we notice that, in three-node relay communication model, the throughput of TRCM-EH-ET is apparently better than that of TRCM-EH although $y_{1}$ and $y_{2}$ are also generated by the same Gamma distribution. The reason is that, in three-node relay communication model, energy transfer depends on not only the relationship between energy harvesting rates $\left(y_{1}\right.$ and $\left.y_{2}\right)$, but also the relationship between channel coefficients $\left(h_{1}\right.$ and $\left.h_{2}\right)$.

Compared with Figure 5, in Figure 7, the throughput of TCM-EH-ET obviously outperform that of TCM-EH, that is because the difference between energy harvesting rates $\left(x_{1}\right.$ and $\left.x_{2}\right)$ is increased, which is caused by different Gamma distributions $\Gamma(8,8)$ and $\Gamma(3,3)$. Compared with Figure 6, Figure 8 shows the similar phenomenon for the same reason.

Figures 9, 10, and 11 show various performances versus energy harvesting rate $x_{1}$ and $x_{2}$ in TCM-EH-ET.

Figure 9 shows that, whether energy harvesting rate $x_{1}$ or $x_{2}$ increases, the throughput of TCM-EH-ET also increases. It demonstrates that higher energy harvesting rate leads to higher throughput performance.

Figure 10 shows that, in TCM-EH-ET, the energy transfer rate $\delta$ becomes high as the gap between $x_{1}$ and $x_{2}$ increases. When $x_{1}$ is close to $x_{2}$, the energy transferred between nodes is reduced to zero. Obviously, according to Equations (14) and (15), when $x_{1}$ is close to $x_{2}$, the energy transfer rate $\delta$ may equal zero. That means the energy transfer may stop even if $x_{1}$ is not equal to $x_{2}$.

In Figure 11, we see that, as the energy harvesting rate $\left(x_{1}\right.$ and $\left.x_{2}\right)$ increases, the TCM-EH-ET tends to reduce the save-ratio. It means that, with higher energy harvesting rate, TCM-EH-ET prefers to leave less time for energy harvesting but more time for data transmission. However, as we discussed above, when $x_{1}$ is close to $x_{2}$, the energy transfer process goes to stop. Thus, the node with lower energy harvesting rate cannot get the energy from the other node and have to increase save-ratio to continue optimizing the throughput performance. Moreover, we also see that there is a drop when $x_{1}$ equals $x_{2}$. That means the optimal throughput performance has been achieved and do not need increase save-ratio. Actually, the optimal throughput performance has been achieved since energy transfer rate $\delta$ equals zero.

Figures 12,13 , and 14 show various performances versus $y_{1} h_{1}$ and $y_{1} h_{2}$ in TRCM-EH-ET.

In Figure 12, we see that, as $y_{1} h_{1}$ or $y_{2} h_{2}$ increases, the throughput of TRCM-EH-ET increases, too. Obviously, bigger energy harvesting rate and better channel coefficients lead to higher throughput.

Figure 13 shows the energy transfer performance in TRCM-EH-ET. The energy transfer rate becomes high as the gap between $y_{1} h_{1}$ or $y_{2} h_{2}$ increases. It means that 
more energy needs to be transferred to improve the throughput performance. Figure 13 also shows that when $x_{1}$ is close to $x_{2}$, the energy transferred between nodes is reduced to zero.

In Figure 14, as the value of $y_{1} h_{1}$ and $y_{2} h_{2}$ increases, TRCM-EH-ET tends to reduce save-ratio. This is because, with higher energy harvesting rate and better channel coefficients, TRCM-EH-ET can allocate more time for data transmission.

\section{Conclusions}

In this paper, we studied energy cooperation based on save-then-transmit protocol in wireless communication system and proposed two schemes (TCM-EH-ET and TRCM-EH-ET) to optimize system throughput. In both models, the nodes which transmit information have no fixed energy supplies and gain energy only via wireless energy harvesting from nature. We utilized convex optimization method and Lagrange multiplier method to solve the optimal save-ratio and energy transfer rate in TCM-EH-ET and TRCM-EH-ET, respectively. The simulation results validated our schemes' advantageous performance in terms of system throughput and also depictured the characteristics of network throughput, transferred energy, and save-ratio. Considering the system throughput is maximized as a sum-throughput, which might lead to a large gap to the individual throughput of each node, therefore, we will focus on the throughput fairness issue in our future work.

\section{Endnotes}

${ }^{\mathrm{a}}$ We adopt the time-division strategy for throughput optimization in both two-node communication model and three-node relay communication model. Note that the second and third time intervals could generally be different for optimal solutions, however, in this paper, we assume they are equal for design simplicity.

${ }^{\mathrm{b}}$ Since the wireless channel condition is complex, for simplicity purpose, some literatures $[22,23]$ used the parameter $h$ to represent the conventional channel coefficient and the parameter $\alpha$ to characterize the energy transfer efficiency, respectively. Here, we also employ the two parameters in our work. As the conventional channel coefficient, $h$ is used to characterize the effect of path loss, shadowing, and multipath fading. As the energy transfer efficiency, $\alpha$ is defined as the ratio of the energy harvested by the receiver over the energy transferred by the transmitter.

${ }^{\mathrm{c}}$ To get the optimal strategy of energy harvesting for the three-node relay communication model, $S$ can harvest energy in both the third and the first phases, since it only sends in the second phase. Thus, its harvested energy can be $0.5(1+\rho T) y_{1}$. Similarly, since $R$ only sends in the third phase, it can harvest energy in both the first and the second phases. Thus, its harvested energy is $0.5(1+\rho T) y_{2}$. However, this optimal strategy will bring relativity to adjacent timeslots, and we will study it in our future work. To simplify the problem, we only focus on the current timeslot that $S$ and $R$ only harvest energy at the first phrase of time interval $(0, \rho T]$ as considered in Section 4.

\section{Appendix}

\section{A. Proof of lemma 2}

The first-order continuous partial derivative of $R_{\text {sum }}(\rho$, $\delta)$ can be expressed as

$$
\begin{aligned}
\frac{\partial R_{\text {sum }}}{\partial \rho} & =-0.5 \log _{2}\left[1+\frac{\rho h\left(x_{1}-\delta\right)}{0.5(1-\rho)}\right]-0.5 \log _{2}\left[1+\frac{p h\left(x_{2}+\alpha \delta\right)}{0.5(1-\rho)}\right] \\
& +\frac{0.5 h\left(x_{1}-\delta\right)}{\ln 2\left[\rho h\left(x_{1}-\delta\right)+0.5(1-\rho)\right]} \\
& +\frac{0.5 h\left(x_{2}+\alpha \delta\right)}{\ln 2\left[\rho h\left(x_{2}+\alpha \delta\right)+0.5(1-\rho)\right]}
\end{aligned}
$$

$$
\begin{aligned}
\frac{\partial R_{\text {sum }}}{\partial \delta}= & -\frac{\rho h(1-\rho)}{\ln 2\left[\rho h\left(x_{1}-\delta\right)-\rho+1\right]} \\
& +\frac{\rho h(1-\rho)}{\ln 2\left[\rho h\left(x_{2}+\alpha \delta\right)-\rho+1\right]}
\end{aligned}
$$

The second-order continuous partial derivative of $R_{\text {sum }}(\rho, \delta)$ can be expressed as

$$
\begin{aligned}
\frac{\partial^{2} R_{\text {sum }}}{\partial \rho \partial \delta} & =\frac{0.5 \rho h}{\ln 2\left[\rho h\left(x_{1}-\delta\right)+0.5(1-\rho)\right]} \\
& -\frac{0.5 \alpha \rho h}{\ln 2\left[\rho h\left(x_{2}+\alpha \delta\right)+0.5(1-\rho)\right]} \\
& -\frac{0.25 h(1-\rho)}{\ln 2(1-\rho)\left[\rho h\left(x_{1}-\delta\right)+0.5(1-\rho)\right]^{2}} \\
& +\frac{0.25 \alpha h(1-\rho)}{\ln 2(1-\rho)\left[\rho h\left(x_{2}+\alpha \delta\right)+0.5(1-\rho)\right]^{2}} \\
\frac{\partial^{2} R_{\text {sum }}}{\partial \rho^{2}} & =-\frac{0.5 h^{2}\left(x_{1}-\delta\right)^{2}}{\ln 2(1-\rho)\left[\rho h\left(x_{1}-\delta\right)+0.5(1-\rho)\right]^{2}} \\
- & \frac{0.5 h^{2}\left(x_{2}+\alpha \delta\right)^{2}}{\ln 2(1-\rho)\left[\rho h\left(x_{2}+\alpha \delta\right)+0.5(1-\rho)\right]^{2}} \\
\frac{\partial^{2} R_{\text {sum }}}{\partial \delta^{2}} & =-\frac{0.5 h^{2} \rho^{2}(1-\rho)}{\ln 2\left[\rho h\left(x_{1}-\delta\right)+0.5(1-\rho)\right]^{2}} \\
- & \frac{0.5 \alpha^{2} h^{2} \rho^{2}(1-\rho)}{\ln 2\left[\rho h\left(x_{2}+\alpha \delta\right)+0.5(1-\rho)\right]^{2}}
\end{aligned}
$$

From Equations (38), (39), and (40), we can derive the following relationship 


$$
\begin{gathered}
A C-B^{2}=\frac{0.25 h_{0}^{4} \rho_{0}^{2}}{(\ln 2)^{2}\left[\rho_{0} h\left(x_{1}-\delta_{0}\right)+0.5(1-\rho)\right]^{4}} \\
\cdot\left[\left(x_{1}-\delta_{0}\right)+\frac{1}{\alpha}\left(x_{2}+\alpha \delta_{0}\right)\right]^{2}>0
\end{gathered}
$$

where $A, B$, and $C$ refer to $\frac{\partial^{2} R_{\text {sum }}}{\partial \rho^{2}} \mid$
and $\frac{\partial^{2} R_{\text {sum }}}{\partial \delta^{2}} \mid\left(\rho_{0}, \delta_{0}\right)$, respectively.

Thus, when $0<\rho<1$ and $A<0$, we have $A C-B^{2}>0$, which proves the validity of lemma 2 .

\section{B. Proof of lemma 3}

The solution of lemma 3 can be derived as

$$
\begin{aligned}
& \ln x=a x+b \\
\Rightarrow & x=e^{a x} e^{b} \\
\Rightarrow & -a x e^{-a x}=-a e^{b} \\
\Rightarrow & x=-\frac{W\left(-a e^{b}\right)}{a}
\end{aligned}
$$

\section{Competing interests}

The authors declare that they have no competing interests.

\section{Acknowledgements}

This work was jointly supported by the Natural Science Foundation of China (No.61440062), the Natural Science Foundation Project of CQ CSTC (No.cstc2012jjA40042), the special fund of Chongqing key laboratory (CSTC), and the program for Changjiang Scholars and Innovative Research Team in University (IRT1299).

\section{Author details}

'Chongqing Key Lab of Mobile Communication Technology, Chongqing University of Posts and Telecommunications, Chongging, China. ${ }^{2}$ Department of Electronics and Communications Engineering, Tampere University of Technology, Tampere, Finland.

Received: 16 September 2014 Accepted: 16 April 2015

Published online: 29 April 2015

\section{References}

1. J Lei, RD Yates, L Greenstein, A generic framework for optimizing single-hop transmission policy of replenishable sensors. IEEE. Trans. Wireless Commun. 8(2), 547-551 (2009)

2. K Huang, Throughput of wireless networks powered by energy harvesting, in Conference Record of the Forty Fifth Asilomar Conference on Signals, Systems and Computers, ASILOMAR, 2011, pp. 8-12

3. K Tutuncuoglu, A Yener, Communicating with energy harvesting transmitters and receivers, in Information Theory and Applications Workshop, ITA, 2012, pp. 240-245

4. J Xu, Y Guo, R Zhang, CoMP meets energy harvesting: a new communication and energy cooperation paradigm, IEEE GLOBECOM, 2013, pp. 2508-2513

5. S Mao, MH Cheung, WS Wong, An optimal energy allocation algorithm for energy harvesting wireless sensor networks (IEEE ICC, Ottawa, ON, 2012), pp. 265-270

6. V Sharma, U Mukherji, V Joseph, S Gupta, Optimal energy management policies for energy harvesting sensor nodes. IEEE. Trans. Wireless Commun. 9(4), 1326-1336 (2010)

7. S Luo, R Zhang, TJ Lim, Optimal save-then-transmit protocol for energy harvesting wireless transmitters. IEEE. Trans. Wireless Commun. 12(3), 1196-1207 (2013)

8. S Yin, E Zhang, J Li, L Yin, L Shufang, Throughput optimization for self-powered wireless communications with variable energy harvesting rate (IEEE WCNC, Shanghai, 2013), pp. 830-835
9. R Zhang, CK Ho, MIMO broadcasting for simultaneous wireless information and power transfer (IEEE GLOBECOM, Houston, TX, USA, 2011), pp. 1-5

10. R Zhang, CK Ho, MIMO broadcasting for simultaneous wireless information and power transfer. IEEE. Trans. Wireless Commun. 12(5), 1989-2001 (2013)

11. Z Ding, SM Perlaza, I Esnaola, HV Poor, Power allocation strategies in energy harvesting wireless cooperative networks. IEEE. Trans. Wireless Commun. 13(2), 846-860 (2014)

12. H Chen, Y Li, Y Jiang, Y Ma, B Vucetic, Distributed power splitting for SWIPT in relay interference channels using game theory. IEEE. Trans. Wireless Commun. 14(1), 410-420 (2015)

13. Z Xiang, M Tao, Robust beamforming for wireless information and power transmission. IEEE Commun. Lett. 1(4), 372-375 (2012)

14. DWK Ng, ES Lo, R Schober, Energy-efficient resource allocation in multiuser OFDM systems with wireless information and power transfer (IEEE WCNC, Shanghai, 2013), pp. 3823-3828

15. L Liu, R Zhang, KC Chua, Wireless information transfer with opportunistic energy harvesting. IEEE. Trans. Wireless Commun. 12(1), 288-300 (2013)

16. MK Watfa, H Al-Hassanieh, S Selman, Multi-hop wireless energy transfer in WSNs. IEEE Commun. Lett. 15(12), 1275-1277 (2011)

17. J Park, B Clerckx, Transmission strategies for joint wireless information and energy transfer in a two-user MIMO interference channel (IEEE ICC, Budapest, 2013), pp. 591-595

18. J Park, B Clerckx, Joint wireless information and energy transfer in a two-user MIMO interference channel. IEEE. Trans. Wireless Commun. 12(8), 4210-4221 (2013)

19. I Krikidis, S Timotheou, S Sasaki, RF energy transfer for cooperative networks: Data relaying or energy harvesting. IEEE Commun. Lett. 16(11), 1772-1775 (2012)

20. AA Nasir, X Zhou, S Durrani, RA Kennedy, Relaying protocols for wireless energy harvesting and information processing. IEEE. Trans. Wireless Commun. 12(7), 3622-3636 (2013)

21. B Gurakan, O Ozel, J Yang, S Ulukus, Energy cooperation in energy harvesting two-way communications (IEEE ICC, Budapest, 2013), pp. 3126-3130

22. B Gurakan, O Ozel, J Yang, S Ulukus, Energy cooperation in energy harvesting communications. IEEE. Trans. Wireless Commun. 61(12), 4884-4898 (2013)

23. K Tutuncuoglu, A Yener, Cooperative energy harvesting communications with relaying and energy sharing (ITW, Information Theory Workshop, 2013). pp. $1-5$

24. RM Corless, On the lambert $w$ function (University of Waterloo, Computer Science Department, Canada, 1993)

25. Department of Applied Mathematics, Tongji University, Advanced mathematics (Higher Education Press, Beijing, 2008)

26. D Melvin, Springer, The algebra of random variables (John Wiley \& Sons Inc, New Jersey, USA, 1979)

\section{Submit your manuscript to a SpringerOpen ${ }^{\odot}$ journal and benefit from:}

- Convenient online submission

- Rigorous peer review

- Immediate publication on acceptance

- Open access: articles freely available online

- High visibility within the field

- Retaining the copyright to your article

Submit your next manuscript at $>$ springeropen.com 